\title{
Computational models of visual neurons specialised in the detection of periodic and aperiodic oriented visual stimuli: bar and grating cells
}

\author{
N. Petkov, P. Kruizinga \\ Centre for High Performance Computing and Institute of Mathematics and Computing Science, University of Groningen, P.O. Box 800, 9700 AV Groningen, \\ The Netherlands
}

Received: 4 June 1996 / Accepted in revised form: 7 October 1996

\begin{abstract}
Computational models of periodic- and aperiodicpattern selective cells, also called grating and bar cells, respectively, are proposed. Grating cells are found in areas $\mathrm{V} 1$ and V2 of the visual cortex of monkeys and respond strongly to bar gratings of a given orientation and periodicity but very weakly or not at all to single bars. This nonlinear behaviour, which is quite different from the spatial frequency filtering behaviour exhibited by the other types of orientation-selective neurons such as the simple cells, is incorporated in the proposed computational model by using an AND-type non-linearity to combine the responses of simple cells with symmetric receptive field profiles and opposite polarities. The functional behaviour of bar cells, which are found in the same areas of the visual cortex as grating cells, is less well explored and documented in the literature. In general, these cells respond to single bars and their responses decrease when further bars are added to form a periodic pattern. These properties of bar cells are implemented in a computational model in which the responses of bar cells are computed as thresholded differences of the responses of corresponding complex (or simple) cells and grating cells. Bar and grating cells seem to play complementary roles in resolving the ambiguity with which the responses of simple and complex cells represent oriented visual stimuli, in that bar cells are selective only for form information as present in contours and grating cells only respond to oriented texture information. The proposed model is capable of explaining the results of neurophysiological experiments as well as the psychophysical observation that the perception of texture and the perception of form are complementary processes.
\end{abstract}

\section{Introduction}

The discovery of orientation-selective cells in the primary visual cortex of monkeys almost 40 years ago and the fact that most of the neurons in this part of the brain are of this type (Hubel and Wiesel 1962, 1974) triggered a wave of

Correspondence to: N. Petkov (e-mail: petkov@cs.rug.nl) research activity aimed at a more precise, quantitative description of the functional behaviour of such cells. Questions of what the optimal stimuli - bars and edges or gratings are for this type of cell and whether the cells carry out bar and edge detection or local frequency analysis gained considerable attention in the literature (Macleod and Rosenfeld 1974; De Valois et al. 1978, 1979; Tyler 1978; Albrecht et al. 1980; von der Heydt 1987). In the meantime functional descriptions and adequate computational models of the main classes of orientation-selective visual neurons such as the simple and complex cells have been proposed and the above questions have received satisfactory answers.

Simple cells can be modelled by linear filters followed by half-wave rectification (Moshvon et al. 1978a; Andrews and Pollen 1979; Maffei et al. 1979; Glezer et al. 1980; Kulikowski and Bishop 1981). Their orientation and spatial frequency selectivity can be explained by the specific kind of linear filtering involved. The space-domain impulse responses of these filters can quite well be approximated by two-dimensional Gabor functions (Daugman 1985; Jones and Palmer 1987) and, knowing the properties of these functions, it is easy to understand why this kind of filter acts as a local edge and bar detector (Petkov 1995). The twodimensional spatial frequency response of such a filter is represented by two Gaussian functions whose centres are symmetrically displaced from the centre of the spatial frequency domain and this explains the orientation and spatial frequency selectivity of the filter and its strong response to gratings of appropriate orientation and periodicity. The above facts, combined with the locality of these filters, explain why they act as local spatial frequency analysers and, at the same time, as local edge and bar detectors. Complex cells behave similarly, but need more intricate modelling which includes three stages: linear filtering, half-wave rectification and local spatial summation (Movshon et al. 1978b; Spitzer and Hochstein 1985; Morrone and Burr 1988; Shapley et al. 1990; Szulborski and Palmer 1990).

The focusing of the attention of the research community on the dilemma of edge/bar detection versus local frequency analysis properties of simple cells may have occluded the functional diversity in the rather broad class of all orientation-selective cells. Relatively recently von der Heydt 
et al. $(1991,1992)$ reported on the discovery of a new type of orientation-selective neuron in areas V1 and V2 of the visual cortex of monkeys which they called grating cells. Similarly to other orientation-selective neurons, such as simple, complex and hyper-complex cells, grating cells respond vigorously to a grating of bars of appropriate orientation, position and periodicity. In contrast to other orientation-selective cells, grating cells respond very weakly or not at all to single bars, i.e., bars which are isolated and do not form part of a grating. This behaviour of grating cells cannot be explained by linear filtering followed by half-wave rectification as in the case of simple cells, neither can it be explained by three-stage models of the type used for complex cells. Most grating cells start to respond when a grating of a few bars (two to five) is presented. In most cases the response rises linearly with the number of additional bars up to a given number (four to 14), after which it quickly saturates and the addition of new bars to the grating causes the response to rise only slightly or not at all and in some cases even to decline. Similarly, the response rises with the length of the bars up to a given length, after which saturation and in some cases inhibition is observed. The responses to moving gratings are unmodulated and do not depend on the direction of movement. The dependence of the response on contrast shows a switching characteristic, in that turn-on and saturation contrast values lie pretty close: the most sensitive grating cells start to respond at a contrast of $1 \%$ and level off at $3 \%$. In general, grating cells are more selective than simple cells, ${ }^{1}$ having spatial frequency bandwidths in the range of 0.4-1.4 octaves, with median 1 octave and orientation bandwidth of about $20^{\circ}$.

During their research on grating cells, von der Heydt et al. (1992) also found other cells which responded to single bars but not at all to square-wave gratings of any periodicity. More generally, this type of cell, which we call bar cells in the following, respond most strongly to single bars and their responses decrease with the addition of further parallel bars to make a grating. In previous studies Schiller et al. (1976) also found many cells in area V1 which responded strongly to single bars and edges but did not respond to sine-wave gratings. Blakemore and Tobin (1972) measured the response of a 'complex' cell to a white bar of optimal orientation, position and size in the presence of a bar grating covering the area outside a circle which was somewhat larger than the region in which the cell responded to the bar stimulus. They observed an inhibition effect due to the grating. This effect was strongest when the grating had the same orientation as the optimal bar stimulus. In this case the response of the cell was reduced to the level of spontaneous activity. The inhibition effect of the grating decreased with the deviation of its orientation from the optimal orientation of the bar stimulus. One may wish to think of this cell as a bar cell similar to the cells described by Schiller et al. and von der Heydt et al. Unfortunately, the properties of this class of cells are not sufficiently well investigated and reported in the literature.

\footnotetext{
${ }^{1}$ Simple cell spatial frequency bandwidths at half response vary in the range $0.4-2.6$ octaves with median 1.4 octaves; their median orientation bandwidth is about $40^{\circ}$ (De Valois et al. 1982).
}

The above properties suggest that the primary role of grating cells is to detect periodicity in oriented patterns, ignoring other details (such as contrast). On the other hand, their higher specialisation and relatively narrow bandwidths cause them to be activated by natural visual stimuli relatively rarely, compared with other orientation-selective cells. The higher specialisation of bar cells compared with other orientation-selective cells raises similar questions. Therefore, the roles of bar and grating cells need to be clarified in order to achieve better insight into the structure of the visual system and the role of functional specialisation. The approach to this problem adopted in this study is a computational one: computational models of bar and grating cells are proposed and used to simulate their activity. On the basis of the results we draw conclusions about the possible role of bar and grating cells in the visual system.

The paper is organised as follows: in Sect. 2 computational models of both simple and complex cells are briefly introduced. These models are well known from the literature, but since they form part of the models of bar and grating cells they are included here for ease of reference and clarity of parametrisation. A computational model of grating cells is given in Sect. 3. In the same section, we present the results of some computer simulations of modelled grating cells. Furthermore grating cell operators are compared with complex cell operators with respect to the detection and segmentation of texture. In Sect. 4 a computational model of bar cells is introduced and the results of computer simulations of such cells, which explain neurophysiological observations, are given. Perceptual experiments are presented and an explanation of the observed phenomena is provided based on the simulations of grating and bar cells using the proposed computational models. In Sect. 5 we summarise the results of the study and draw some conclusions about the role which grating and bar cells play in the processing of visual information.

\section{Preliminary: computational models of simple and complex cells}

Bar and grating cells are found in the same cortical area (V1) as simple and complex cells and similarly to simple and complex cells show orientation selectivity. On the other hand they show a more complex non-linear behaviour and a sharper orientation and spatial frequency tuning. These facts suggest that bar and grating cells receive input from simple or complex cells and below we propose a model in which the responses of simple and complex cells are used to compute the responses of bar and grating cells. This is similar to the idea that complex cells may receive inputs from simple cells (Hubel 1982) - an idea which explicitly or implicitly is used in most models of complex cells. Since simple cells play a substantial role in the following, we first briefly introduce a computational model of this type of cell. The response $r$ of a simple cell which is characterised by a receptive field function $g(x, y)$ to a luminance distribution image $f(x, y),(x, y) \in \Omega$, is computed as follows ( $\Omega$-visual field domain):

$r=\chi\left(\iint_{\Omega} f(x, y) g(x, y) \mathrm{d} x \mathrm{~d} y\right)$ 
where $\chi$ is the Heaviside step function $(\chi(z)=0$ for $z<0$, $\chi(z)=z$ for $z \geq 0$ ). Below we extend this simple model with a local contrast compensation.

We use the following family of two-dimensional Gabor functions (Daugman 1985) to model the spatial summation properties of simple cells: ${ }^{2}$

$$
\begin{aligned}
g_{\xi, \eta, \lambda, \Theta, \varphi}(x, y) & =\mathrm{e}^{-\frac{\left(x^{\left.\prime^{2}+\gamma^{2} y^{2}\right)}\right.}{2 \sigma^{2}} \cos \left(2 \pi \frac{x^{\prime}}{\lambda}+\varphi\right)} \\
x^{\prime} & =(x-\xi) \cos \Theta-(y-\eta) \sin \Theta \\
y^{\prime} & =(x-\xi) \sin \Theta+(y-\eta) \cos \Theta,
\end{aligned}
$$

where the arguments $x$ and $y$ specify the position of a light impulse in the visual field and $\xi, \eta, \sigma, \gamma, \lambda, \Theta$ and $\varphi$ are parameters as follows:

The pair $(\xi, \eta)$, which has the same domain $\Omega$ as the pair $(x, y)$, specifies the centre of a receptive field within the visual field. The standard deviation $\sigma$ of the Gaussian factor determines the (linear) size of the receptive field. Its eccentricity and herewith the eccentricity of the receptive field ellipse is determined by the parameter $\gamma$, called the spatial aspect ratio. It has been found to vary in a limited range of $0.23<\gamma<0.92$ (Jones and Palmer 1987). The value $\gamma=0.5$ is used in our simulations and, since this value is constant, the parameter $\gamma$ is not used to index a receptive field function.

The parameter $\lambda$ is the wavelength and $1 / \lambda$ the spatial frequency of the harmonic factor $\cos \left(2 \pi x^{\prime} / \lambda+\varphi\right)$. The ratio $\sigma / \lambda$ determines the spatial frequency bandwidth ${ }^{3}$ of simple cells and thus the number of parallel excitatory and inhibitory stripe zones which can be observed in their receptive fields. Neurophysiological research has shown that the half-response spatial-frequency bandwidths of simple cells vary in the range of $0.5-2.5$ octaves in the cat (Movshon et al. 1978a; Albrecht et al. 1979; Andrews and Pollen 1979; Kulikowski and Bishop 1981) [weighted mean 1.32 octaves (Daugman 1985)] and 0.4-2.6 octaves in the macaque monkey (De Valois et al. 1982) (median 1.4 octaves). While there is a considerable spread, the bulk of cells have bandwidths in the range 1.0-1.8 octaves. De Valois et al. (1982) proposed that this spread is due to the gradual sharpening of the orientation and spatial frequency bandwidth at consecutive stages of the visual system and that the input to higher processing stages is provided by the more narrowly tuned simple cells with half-response spatial frequency bandwidth of approximately one octave. This value of the half-response spatial frequency bandwidth corresponds to the value 0.56 of the ratio $\sigma / \lambda$ which is used in the simulations of this study. Since $\lambda$ and $\sigma$ are not independent $(\sigma / \lambda=0.56)$, only one of them is considered as a free parameter which is used to index a receptive field function. For ease of reference to the spatial frequency properties of the cells, we choose $\lambda$ to be this free parameter.

The angle parameter $\Theta(\Theta \in[0, \pi))$ specifies the orientation of the normal to the parallel excitatory and inhibitory

\footnotetext{
${ }^{2}$ Our modification of the parametrisation used in Daugman (1985) takes into account the restrictions found in experimental data.

${ }^{3}$ The half-response spatial frequency bandwidth $b$ (in octaves) of a linear filter with an impulse response according to (2) is the following function of the ratio $\sigma / \lambda: b=\log _{2} \underset{\lambda}{\underset{\lambda}{\sigma}+\frac{1}{\pi} \sqrt{\frac{1}{2}} \sqrt{\frac{\ln 2}{2}} \sqrt{\ln 2}}$. Inversely, $\underset{\lambda}{\sigma}=\frac{1}{\pi} \sqrt{\frac{\ln 2}{2} \cdot 2^{b}-1}$.
}

stripe zones - this normal is the axis $x^{\prime}$ in (2) - which can be observed in the receptive fields of simple cells. ${ }^{4}$

Finally, the parameter $\varphi(\varphi \in(-\pi, \pi])$, which is a phase offset in the argument of the harmonic factor $\cos \left(2 \pi x^{\prime} / \lambda+\varphi\right)$, determines the symmetry of the function $g_{\xi, \eta, \lambda, \Theta, \varphi}(x, y)$ : for $\varphi=0$ and $\varphi=\pi$ it is symmetric, or even, with respect to the centre $(\xi, \eta)$ of the receptive field; for $\varphi=-{ }_{2}^{1} \pi$ and $\varphi={ }_{2}^{1} \pi$, the function is antisymmetric, or odd, and all other cases are asymmetric mixtures of these two. In our simulations we use for $\varphi$ the following values: $\varphi=0$ for symmetric receptive fields to which we refer as 'centre-on' in analogy with retinal ganglion cell receptive fields whose central areas are excitatory; $\varphi=\pi$ for symmetric receptive fields to which we refer to as 'centre-off' since their central lobes are inhibitory; and $\varphi=-{ }_{2}^{1} \pi$ and $\varphi={ }_{2}^{1} \pi$ for antisymmetric receptive fields with opposite polarities. [There are certain arguments in support of this choice based on the results of psychophysical (Field and Nachmias 1984; Burr et al. 1989) and neurophysiological (De Valois et al. 1978; Movshon et al. 1978a; Kulikowski and Bishop 1981) experiments. Other neurophysiological studies suggest that asymmetric receptive fields exist as well (Hubel and Wiesel 1962; Andrews and Pollen 1979; Marcelja 1980; Field and Tolhurst 1986) or even that the distribution of phases is uniform (Daugman 1985). A remarkable finding is the existence of pairs of nearby cells with phase difference of ${ }_{2}^{1} \pi$ (Pollen and Ronner 1981).] Intensity map illustrations of receptive field functions of different positions, sizes, orientations and symmetries are shown in Fig. 1.

As to the importance of simple cells for the visual system, it is believed that they play a significant role in the process of form perception, in that they act as detectors of oriented intensity transitions such as edges and bars. More specifically, a cell with a symmetric receptive field will react strongly (but not exclusively) to a bar which coincides in direction, width and polarity with the central lobe of the receptive field. A cell with an antisymmetric receptive field will react strongly (but also not exclusively) to an edge of the same orientation if the excitatory lobe is on the light side of the transition and the inhibitory lobe on its dark side. As to the spatial frequency selectivity of simple cells, Fig. 2 illustrates the spatial frequency responses which correspond to the receptive fields shown in Fig. 1. The light areas indicate spatial frequencies and wavevector orientations which will be passed by such filters; all other wave components will be rejected or strongly attenuated. These spatial frequency responses explain the selectivity of simple cells for gratings of appropriate orientation and periodicity.

Using the above parametrisation, one can compute the response $s_{\xi, \eta, \lambda, \Theta, \varphi}$ of a simple cell modelled by a receptive field function $g_{\xi, \eta, \lambda, \Theta, \varphi}(x, y)$ to an input image with luminance distribution $f(x, y)$ as follows:

First, an integral

$$
r_{\xi, \eta, \lambda, \Theta, \varphi}=\iint_{\Omega} f(x, y) g_{\xi, \eta, \lambda, \Theta, \varphi}(x, y) \mathrm{d} x \mathrm{~d} y
$$

\footnotetext{
${ }^{4}$ Typically three to five parallel excitatory and inhibitory stripe zones can be observed in the receptive fields of simple cells, depending on their spatial frequency bandwidths.
} 


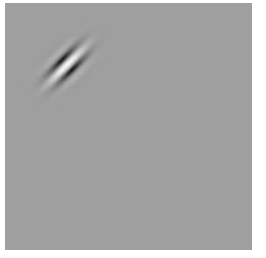

a

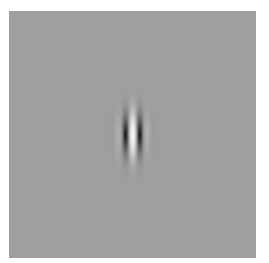

e
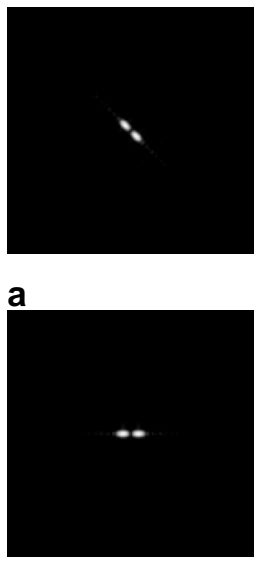

e
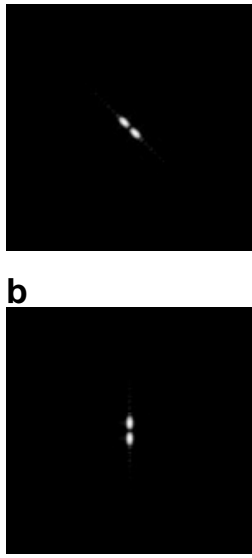

f

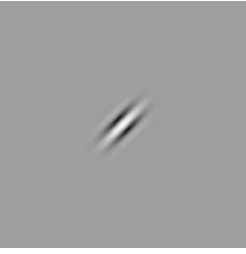

b

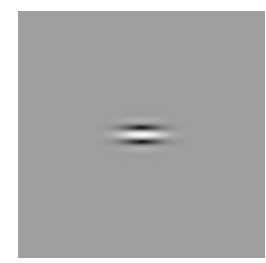

f
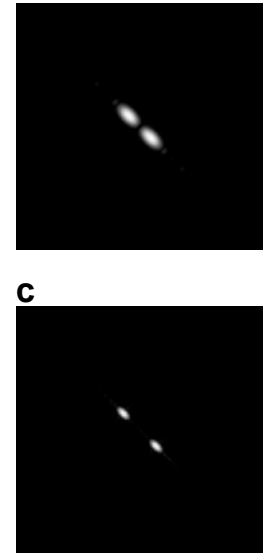

g

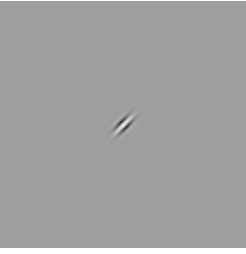

C

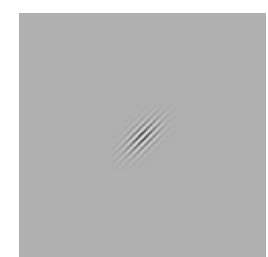

g

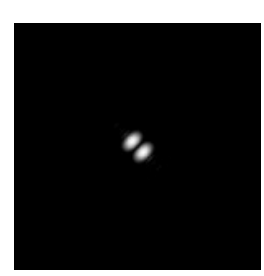

d

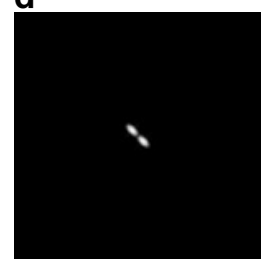

h

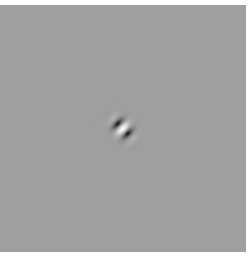

d

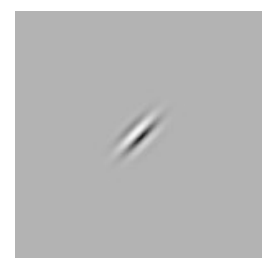

h

Fig. 1. Receptive fields of different positions (a,b), sizes (b,c), eccentricities (b,d), orientations (e,f), number of excitatory and inhibitory zones $(\mathbf{b}, \mathbf{g})$, and symmetries $(\mathbf{b}, \mathbf{h})$. Grey levels which are lighter and darker than the background indicate excitatory and inhibitory zones, i.e. zones in which the function takes positive and negative values, respectively

Fig. 2. Power spectra of the receptive field functions shown in Fig.1

is evaluated in the same way as though the receptive field function $g_{\xi, \eta, \lambda, \Theta, \varphi}(x, y)$ were the response of a linear system. In order to normalise the simple cell response with respect to the contrast of the input image, $r_{\xi, \eta, \lambda, \Theta, \varphi}$ is divided by the average grey level within the receptive field. The average $a_{\xi, \eta, \lambda}$ is computed using the Gaussian factor of the function $g_{\xi, \eta, \lambda, \Theta, \varphi}$ :

$a_{\xi, \eta, \lambda}=\iint_{\Omega} f(x, y) \mathrm{e}^{-\frac{(x-\xi)^{2}+\gamma^{2}(y-\eta)^{2}}{2 \sigma^{2}}} \mathrm{~d} x \mathrm{~d} y$

The ratio $r_{\xi, \eta, \lambda, \Theta, \varphi} / a_{\xi, \eta, \lambda}$ is proportional to the local contrast within the receptive field of a cell. In order to obtain a contrast response function similar to the ones measured on real cells, we use the hyperbolic ratio function to calculate the simple cell response from the ratio $\frac{r_{\xi, \eta, \lambda, \Theta, \varphi}}{a_{\xi, \eta, \lambda}}$.

$s_{\xi, \eta, \lambda, \Theta, \varphi}= \begin{cases}0 & \text { if } a_{\xi, \eta, \lambda}=0 \\ \chi\left(\begin{array}{c}r_{\xi, \eta, \lambda, \Theta, \varphi} \\ a_{\xi, \eta, \lambda} \\ r_{\xi, \eta, \lambda, \Theta, \varphi}+C \\ a_{\xi, \eta, \lambda}+C\end{array}\right) & \text { otherwise }\end{cases}$

where $R$ and $C$ are the maximum response level and the semi-saturation constant, respectively.

In the following we also need a computational model of complex cells for a comparison of their computed responses to oriented texture with the computed responses of grating cells and as input to bar cell operators. We use the following model of complex cells:

$c_{\xi, \eta, \lambda, \Theta}=\iint_{\Omega} \mathrm{e}^{-\frac{\left(\xi-\xi^{\prime}\right)^{2}+\gamma^{\prime}\left(\eta-\eta^{\prime}\right)^{2}}{2\left(\sigma^{\prime}\right)^{2}}} \sqrt{\sum_{\varphi} s_{\xi^{\prime}, \eta^{\prime}, \lambda, \Theta, \varphi}^{2}} \mathrm{~d} \xi^{\prime} \mathrm{d} \eta^{\prime}$

which represents weighted spatial summation of the quadrature responses of simple cells of the same preferred orientation $\Theta$ and spatial frequency $1 / \lambda$, but with receptive field centres $\left(\xi^{\prime}, \eta^{\prime}\right)$ spread within the neighbourhood of the receptive field centre $(\xi, \eta)$ of the complex cell. The size of this neighbourhood is determined by the parameter $\sigma^{\prime}$, which we choose to be two times greater than the respective parameter $\sigma$ in the model of the simple cell, $\sigma^{\prime}=2 \sigma$. We have to note that this model describes only one type of cell in the rather broad class of complex cells. Complex cells of this type will respond to edges and bars of appropriate orientation within their receptive fields, regardless of their exact position and polarity (i.e. there is no phase modulation). This model is sufficient for the purpose of this study. 


\section{Grating cells}

\subsection{Computational model}

Von der Heydt et al. (1991) have proposed a model of grating cells in which the activities of displaced semi-linear units of the simple cell type are combined by an AND-type nonlinearity to produce grating cell activity. While the simple model they propose reacts to gratings of appropriate orientation and periodicity and does not react to single bars, it will also react to a number of stimuli to which grating cells would not respond, such as a bar and an edge parallel to it. Their simple model also does not account for correct spatial frequency tuning - it will for instance react not only to the fundamental spatial frequency but also to all multiples of it - and the spatial summation properties of grating cells. We therefore give an alternative model of grating cells which is aimed at reproducing all their properties which are known from neurophysiological experiments.

Our model of grating cells consists of two stages (Kruizinga and Petkov 1995). In the first stage, the responses of so-called grating subunits are computed using as input the responses of centre-on and centre-off simple cells with symmetric receptive fields. The model of a grating subunit is conceived in such a way that the unit is activated by a set of three bars with appropriate periodicity, orientation and position. In the next, second stage, the responses of grating subunits of a given preferred orientation and periodicity are summed together within a certain area to compute the response of a grating cell. This model is next explained in more detail.

A quantity $q_{\xi, \eta, \Theta, \lambda}$, called the activity of a grating subunit with position $(\xi, \eta)$, preferred orientation $\Theta$ and preferred grating periodicity $\lambda$, is computed as follows:

$q_{\xi, \eta, \Theta, \lambda}=\left\{\begin{aligned} & 1 \text { if } \quad \forall n, n \in\{-3 \ldots 2\}, \\ & M_{\xi, \eta, \Theta, \lambda, n} \geq \rho \mathcal{M}_{\xi, \eta, \Theta, \lambda} \\ & 0 \text { if } \begin{array}{l}\exists, n \in\{-3 \ldots 2\}, \\ \\ M_{\xi, \eta, \Theta, \lambda, n}<\rho \mathcal{M}_{\xi, \eta, \Theta, \lambda}\end{array}\end{aligned}\right.$

where $\rho$ is a threshold parameter with a value smaller than but near 1 (e.g. $\rho=0.9$ ) and the auxiliary quantities $M_{\xi, \eta, \Theta, \lambda, n}$ and $\mathcal{M}_{\xi, \eta, \Theta, \lambda}$ are computed as follows:

$$
\begin{aligned}
& M_{\xi, \eta, \Theta, \lambda, n}=\max \left\{s_{\xi^{\prime}, \eta^{\prime}, \Theta, \lambda, \varphi_{n}} \mid \xi^{\prime}, \eta^{\prime}:\right. \\
& { }_{n}{ }_{2}^{\lambda} \cos \Theta \leq\left(\xi^{\prime}-\xi\right)<(n+1) \frac{\lambda}{2} \cos \Theta, \\
& { }_{2}^{\lambda} \sin \Theta \leq\left(\eta^{\prime}-\eta\right)<(n+1) \frac{\lambda}{2} \sin \Theta \text {, }
\end{aligned}
$$

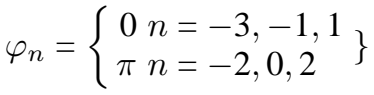

and

$\mathcal{M}_{\xi, \eta, \Theta, \lambda}=\max \left\{M_{\xi, \eta, \Theta, \lambda, n} \quad \mid \quad n=-3 \ldots 2\right\}$

The quantities $M_{\xi, \eta, \Theta, \lambda, n}, n=-3 \ldots 2$, are related to the activities of simple cells with symmetric receptive fields along a line segment of length $3 \lambda$ passing through point $(\xi, \eta)$ in orientation $\Theta$. This segment is divided in intervals of length $\lambda / 2$ and the maximum activity of one sort of simple cell, centre-on or centre-off, is determined in each interval. a)

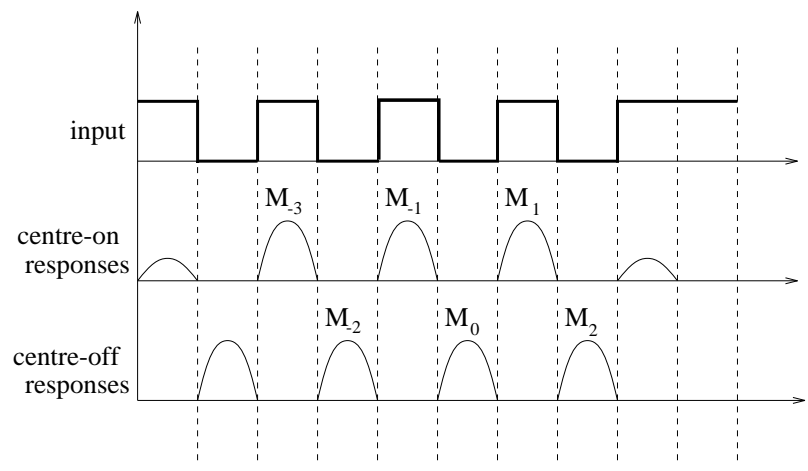

Fig. 3. Luminance distribution along a normal to a set of three square bars (a), and the distribution of the computed responses of centre-on (b) and centre-off (c) cells along this line

$M_{\xi, \eta, \Theta, \lambda,-3}$, for instance, is the maximum activity of centreon simple cells in the corresponding interval of length $\lambda / 2$; $M_{\xi, \eta, \Theta, \lambda,-2}$ is the maximum activity of centre-off simple cells in the adjacent interval, etc. Centre-on and centre-off simple cell activities are alternately used in consecutive intervals. $\mathcal{M}_{\xi, \eta, \Theta, \lambda}$ is the maximum among the above interval maxima.

Roughly speaking, a grating cell subunit will be activated if centre-on and centre-off cells of the same preferred orientation $^{5} \Theta$ and spatial frequency $1 / \lambda$ are alternately activated in intervals of length $\lambda / 2$ along a line segment of length $3 \lambda$ centred on point $(\xi, \eta)$ and passing in direction $\Theta$. This will, for instance, be the case if three parallel bars with spacing $\lambda$ and orientation $\Theta$ of the normal to them are encountered (Fig. 3). In contrast, the condition is not fulfilled by the simple cell activity pattern caused by only a single bar or two bars.

At this point, the question might be raised as to why this condition is applied to the responses of simple cells and not to the pixel values of the input image. If applied to the pixels of the input image, periodicity of three crests and three troughs along a line with orientation $\Theta$ passing through point $(\xi, \eta)$ will be detected. This periodicity need not, however, be due to a system of three parallel bars. Experiments with checkerboard patterns [see fig. 12D in von der Heydt et al. (1992)] in which the direction of the periodicity of the checks does not coincide with the normal to the diagonals this is the case when the aspect ratio of the checks is different from 1 - have shown that grating cells detect the periodicity of the diagonals (which evidently resemble bars in the response they elicit) rather than the periodicity of the checks. Simple cells with spatial aspect ratio $\gamma<1$ have elongated excitatory and inhibitory zones which will integrate the luminance distribution over more than one check leading to a small overall response. It is this integration which simple cells carry out in the excitatory and inhibitory stripe zones of their receptive fields, which provides that grating cells will react to patterns of appropriately oriented bars but will not react to periodic point and checkerboard patterns.

In the next, second stage of the model, the response $w_{\xi, \eta, \Theta, \lambda}$ of a grating cell whose receptive field is cen-

\footnotetext{
${ }^{5}$ Note that with respect to the orientation of receptive fields, the parameter $\Theta$ specifies the normal to the system of parallel excitatory and inhibitory regions.
} 

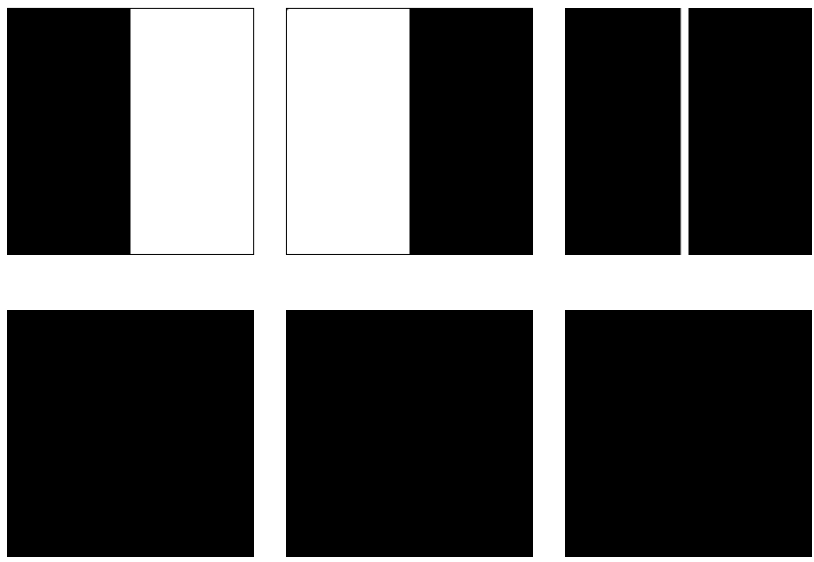

b

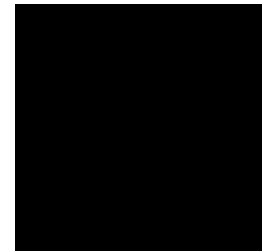

C
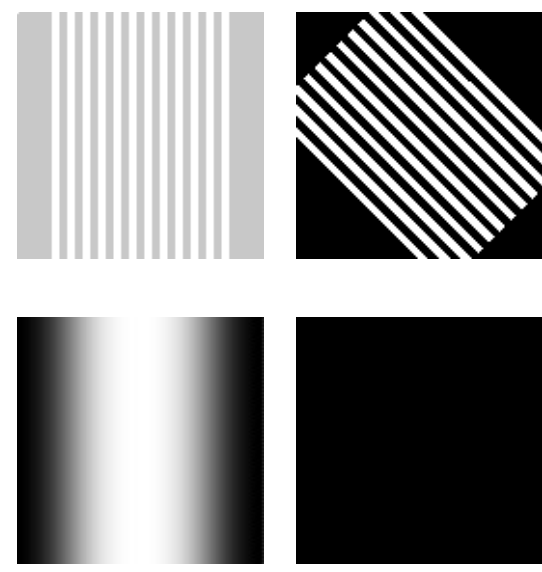

b

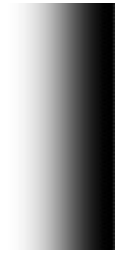

a

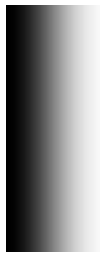

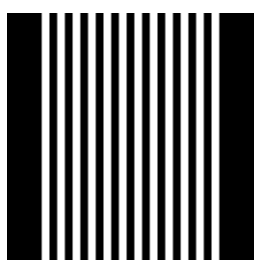

tred on point $(\xi, \eta)$ and which has a preferred orientation $\Theta(\Theta \in[0, \pi))$ of the normal to the grating and periodicity $\lambda$ is computed by weighted summation of the responses of the grating subunits. At the same time the model is made symmetric for opposite directions by taking the sum of grating subunits with orientations $\Theta$ and $\Theta+\pi$.

$$
w_{\xi, \eta, \Theta, \lambda}=\int \mathrm{e}^{-\frac{\left(\xi-\xi^{\prime}\right)^{2}+\left(\eta-\eta^{\prime}\right)^{2}}{2(\beta \sigma)^{2}}}\left(q_{\xi^{\prime}, \eta^{\prime}, \Theta, \lambda}+q_{\xi^{\prime}, \eta^{\prime}, \Theta+\pi, \lambda}\right) \mathrm{d} \xi^{\prime} \mathrm{d} \eta^{\prime},
$$$$
\Theta \in[0, \pi)
$$

The weighted summation is a provision made to model the spatial summation properties of grating cells with respect to the number of bars and their length as well as their unmodulated responses with respect to the exact position (phase) of a grating. The parameter $\beta$ determines the size of the area over which effective summation takes place. A value of $\beta=5$ results in a good approximation of the spatial summation properties of grating cells.

\subsection{Computer simulations of grating cell experiments}

Von der Heydt et al. (1992) describe the responses of grating cells to different visual stimuli. We next turn to the question of how the computational model presented above performs for the set of visual stimuli used by von der Heydt et al. in

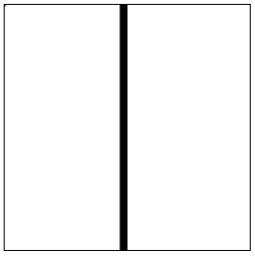

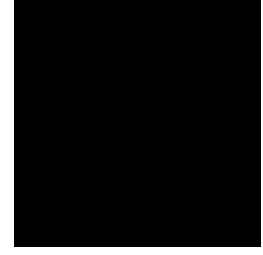

d
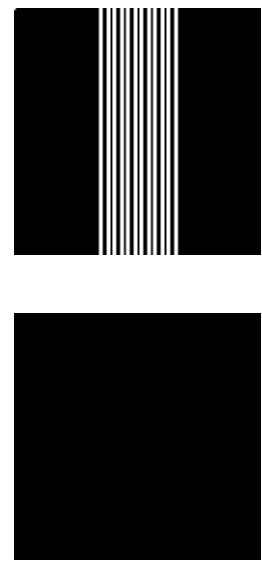

d
Fig. 4a-d. Input visual stimuli (upper row) and computed feature images which correspond to grating cell responses (lower row). None of the cells is activated (black and white mean no activity and strong activity, respectively). The simulated grating cells have vertical preferred orientation, $\Theta=0$, and periodicity of $\lambda=0.03125 L$ (where $L$ is image size)

Fig. 5a-d. Input visual stimuli (upper row) and computed feature images which correspond to the computed grating cell responses (lower row). The simulated grating cells have vertical preferred orientation (orientation $\Theta=0$ of the normal to the optimal grating), and periodicity of $\lambda=0.03125 L$ (where $L$ is image size). Simulated grating cells respond vigorously to a grating of appropriate orientation and periodicity, regardless of contrast $(\mathbf{a}, \mathbf{b})$ but are not activated by high-contrast gratings in which either the orientation differs substantially from the optimal stimulus orientation (c) or the periodicity of the grating pattern is disturbed (d) their experiments. The aim is to validate the model and to find the values of its parameters $(\rho$ and $\beta$ ) for which it will optimally approximate the behaviour of grating cells.

In Fig. 4 the upper row of images shows a set of input visual stimuli for which the responses computed according to the model presented above are visualised in the respective images of the lower row. This presentation form of computed grating cell responses needs an explanation, since it differs from the one used by von der Heydt et al. (1992) to illustrate the results of their neurophysiological experiments (compare their fig. 1). The intensity of a point $(\xi, \eta)$ in an image of the lower row of Fig. 4 represents the computed activity $w_{\xi, \eta, \Theta, \lambda}$ of a grating cell with preferred orientation $\Theta$ (of the normal to the optimal grating), periodicity $\lambda$ and a receptive field centred at point $(\xi, \eta)$. The computed activities of the grating cells which have the same preferred orientation $\Theta$ and periodicity $\lambda$ but differ in the position of their receptive fields are thus represented together in one image. (Such images are referred to as feature images in image processing and computer vision.)

In the particular case shown in Fig. 4, grating cells with vertical preferred orientation are simulated; although the oriented stimuli in the input images have the same orientation as the preferred orientation of the cells, and although they have enough spectral power in the spatial frequency domain for which the cells are selective, none of the cells is activated 


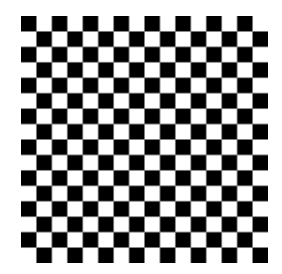

a

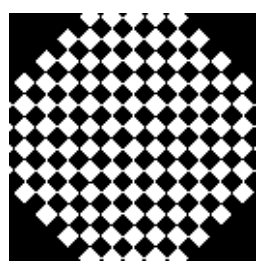

a

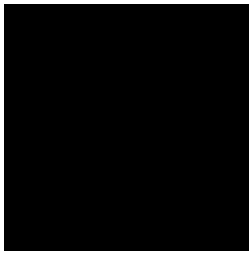

b

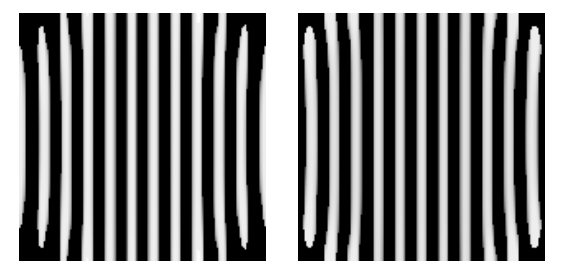

b

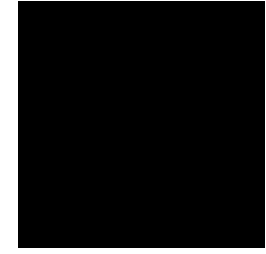

C

C

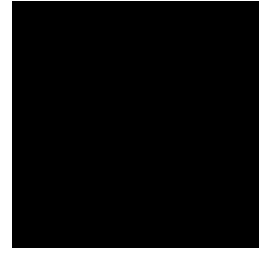

d

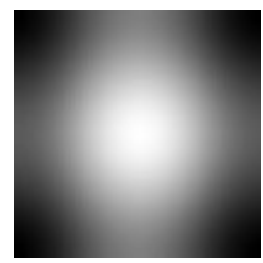

d
Fig. 6. A checkerboard input stimulus (a) and a feature image (d) comprising the responses of simulated grating cells with vertical preferred orientation and preferred periodicity equal to the periodicity of the checkerboard in horizontal orientation. The middle images are the corresponding feature images of centreon (b) and centre-off (c) simple cell responses used to compute the feature image on the right (d)

Fig. 7. A rotated checkerboard input pattern (a) and a feature image (d) comprising the responses of simulated grating cells with vertical preferred orientation and preferred periodicity equal to the periodicity of the checkerboard diagonals in horizontal orientation. The feature images shown in $\mathbf{b}$ and $\mathbf{c}$ show the corresponding centre-on and centre-off simple cell responses, respectively, used to compute the grating cell responses in the feature on the right (d) by edges or single bars. In contrast, many cells are activated by a grating of bars with the proper orientation and periodicity as illustrated by Fig. 5a,b. Bar gratings of orientation and periodicity which differ substantially from the preferred orientation and periodicity of the simulated grating cells fail to activate them, as illustrated by Fig. 5c and Fig. 5d, respectively.

Figure 6 illustrates the behaviour of the grating cell model when a checkerboard pattern (Fig. 6a) is presented. In this simulation a model of grating cells with vertical preferred orientation $(\Theta=0)$ and periodicity $\lambda$ equal to the periodicity of the checkerboard in horizontal orientation is used. The simulated cells would respond to one isolated row of checks but, as can be seen from Fig. 6c, the cells do not respond when the checkerboard pattern is presented as a whole. [Real grating cells do not respond in this case either: see fig. 12B in von der Heydt et al. (1992).] This is due to the fact that the simple cells whose responses are used in the model integrate the intensity along the columns of the checkerboard in both the excitatory and inhibitory regions of their receptive fields and are not activated as shown in Fig. 6b. In this way the model is made sensitive for periodicity of bar gratings but not to mere periodicity along a line.

Figure 7 illustrates the behaviour of the grating cell model when a rotated checkerboard pattern (Fig. 7a) is presented. A model of grating cells with vertical preferred orientation $(\Theta=0)$ and periodicity $\lambda$ equal to the periodicity of the diagonals of the checkerboard in horizontal orientation is used. Similar to their biological counterparts [compare with fig. 12D in von der Heydt et al. (1992)] the simulated grating cells detect the periodicity of the diagonals, although perceptually one may rather give preference to the periodicity along the rows and columns.

As illustrated by the above computer simulation experiments, the proposed model is capable of qualitatively reproducing all important properties of grating cells as reported in von der Heydt et al. (1992). By means of choosing the values of the parameters of the model, we were able to reproduce quantitative properties of grating cells, such as orientation bandwidth of $22.5^{\circ}$ and spatial frequency bandwidth of 1.1 octaves. [It should be noted that similar to simple cells, grating cells show a considerable spread in their orientation and spatial frequency bandwidths. Since we use responses of simple cells with fixed orientation and spatial frequency bandwidths (of $38.6^{\circ}$ and 1.0 octaves, respectively), we obtain a model of grating cells in which the resulting orientation and spatial frequency bandwidths are fixed too.]

\subsection{Detection of oriented texture by grating cells}

Since the grating cell operators introduced above are selective for periodic oriented patterns such as bar gratings, one may expect that they can more generally be used to detect oriented texture. Other orientation-selective operators, such as simple and complex cell operators, have already been shown to be capable of detecting oriented texture in various computer simulation studies (Malik and Perona 1990; Bergen and Landy 1991; Manjunath and Chellappa 1993). It is therefore interesting to consider the question of what the 'added value' of grating cells might be with respect to the detection of oriented texture.

Figure 8 a shows an oriented texture pattern to which two types of filters are applied: one based on complex cells and the other on grating cells. The feature image shown in Fig. $8 \mathrm{~b}$ is computed as a max-value pixel-wise superposition of feature images computed with complex cell operators of different preferred orientations and spatial frequencies. The feature image shown in Fig. 8c is a similar superposition based on grating cell operators. One can conclude that both types of operators are capable of detecting oriented texture, giving comparable results.

The case shown in Fig. 9a is more complex, since several texture regions of different characteristic orientations and periodicities are involved. In this case, the question is whether the two types of orientation and spatial frequency selective operators succeed in segmenting the texture regions. As illustrated by Fig. 9b and Fig. 9c the results are comparable in this case also.

Figure 10 illustrates the results of the application of the same operators on an input image which contains no texture at all. The feature image computed as a max-value superposition of feature images obtained from complex cell operators with different preferred orientations and spatial fre- 


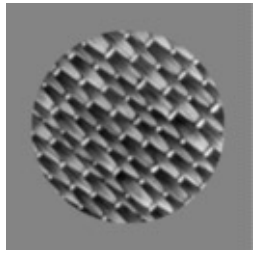

a

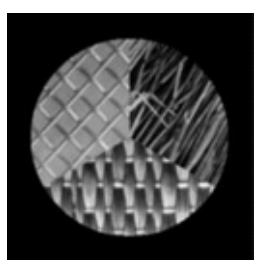

a

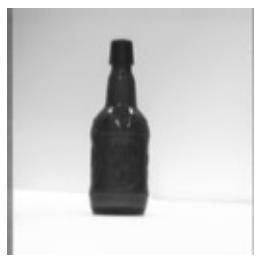

a

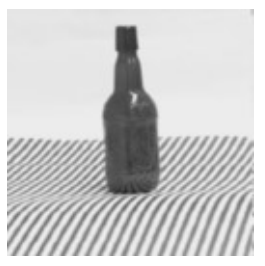

a

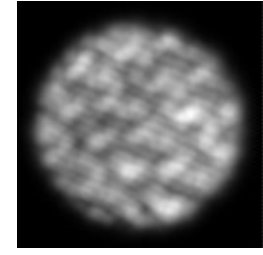

b

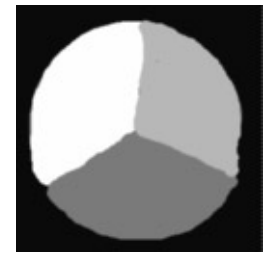

b

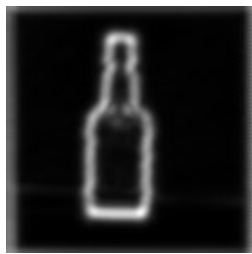

b

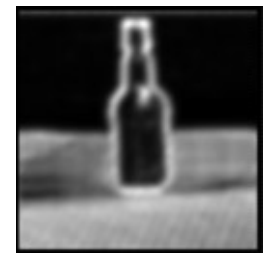

b

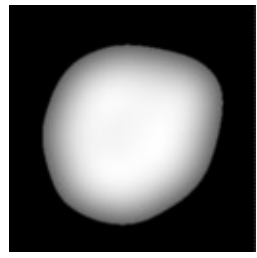

C

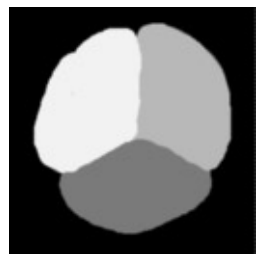

C

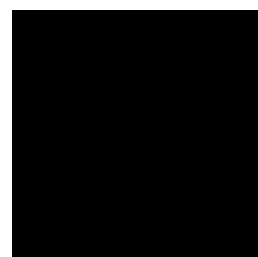

C

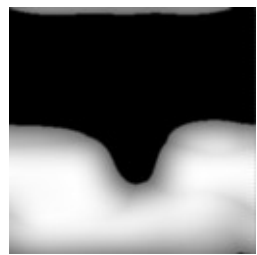

C
Fig. 8. The oriented texture in the input image (a) is detected by both complex (b) and grating (c) cell operators

Fig. 9. A texture input image (a) and computed complex (b) and grating (c) cell feature images. Different shadings are used to render areas with different characteristic profiles of the activity distribution across the different orientation and spatial frequency channels. The regions are uniform, since vector quantisation was applied

Fig. 10. While complex cell operators (b) detect features, such as edges, in an input image (a) which contains no (oriented) texture, grating cell operators (c) do not respond to non-texture image attributes

Fig. 11. While complex cell operators (b) detect both texture and contours in the input image (a), grating cell operators (c) detect only texture and do not respond to other image attributes, such as contours quencies contains features (Fig. 10b). In this particular case the detected features correspond to the edges of the object which is present in the input image. This operator, which was shown above to detect oriented texture quite successfully (Fig. 8b), evidently responds not only to texture but to other image attributes as well. In fact this drawback is common to virtually all operators used for texture analysis in image processing and computer vision: while a specific operator can be developed for the reliable detection of any given texture pattern, the operator will certainly react not exclusively to this texture pattern but also to a number of other patterns as well, even to those which are not perceived as texture at all. In contrast to the complex cell operator, the corresponding grating cell operator detects no features in this case (Fig. 10c). In this way grating cell operators fulfil a very important requirement imposed on texture operators in that next to successfully detecting (oriented) texture, they do not react to other image attributes such as object contours.

Finally, Fig. 11 illustrates the effect of the chosen complex and grating cell operators on images which contain both texture and form information. While the complex cell operator detects both contours and texture and is, in this way, not capable of discriminating between these two different types of image features, the grating cell operator detects ex- clusively (oriented) texture. We conclude that grating cell operators are more effective than (simple and) complex cell operators in the detection of texture in that they are capable not only of detecting texture but also of separating it from other image features, such as object edges and contours.

\section{Bar cells}

\subsection{Influence of texture on the perception of form}

In the computational model of grating cells introduced above the outputs of simple cell operators are used as inputs to grating cell operators. In this way the activities of the former determine the activities of the latter. The relation between simple and grating cell operators can also be considered in the opposite direction. More specifically, in the following we introduce a mechanism in which the activities of grating cell operators can influence the way in which the activities of simple and complex cell operators are conveyed to higher stages, in particular those stages which are concerned with form as represented by edges and contours of objects. This model is capable of explaining the influence of texture on the perception of form, the basic assumptions being that oriented 
texture is detected by grating cells and that the activities of these cells control the process of forwarding the form information encoded in the activities of simple and complex cells to higher stages of form analysis.

We start with a psychophysical experiment which illustrates the influence of oriented texture on the perception of form. Figure 12a shows an image which contains a bar grating of given orientation and periodicity filling a circular region. Superimposed on this grating are two bars with different orientations. These two bars and one of the grating bars form a triangle which is clearly seen if the other bars of the grating are removed (Fig. 12b). In the presence of the grating, however, this triangle does not 'pop out'. The third side of the triangle is quite well perceived as a bar in the grating but - unless special attention is paid to it - it is not perceived as a part of the contour of a triangle. This effect can be observed even if the contrast of this bar is different by a quite considerable extent from the contrast of the other bars of the grating. (In this respect one could turn around the saying 'you cannot see the wood for the trees' into 'you cannot see the tree for the wood'.) In other words, the bar is perceived as a part of the texture but not as an attribute of form, such as a part of the contour of an object. If asked to describe the image in Fig. 12a, one is more likely to say that one sees a grating and two lines of a different orientation (a decomposition shown in Fig. 12c,d) rather than a grating and a triangle (Fig. 12e,f) ${ }^{6}$ We next introduce a computational model which explains this perceptual effect.

\subsection{Computational model of bar cells}

Let $c_{\xi, \eta, \lambda, \theta}$ denote the activity of a complex cell operator whose receptive field is centred on a point $(\xi, \eta)$, has a preferred spatial frequency $1 / \lambda$ and preferred orientation $\theta$. Let $w_{\xi, \eta, \lambda, \theta}$ be the activity of a grating cell operator whose parameters have a similar meaning. We now introduce a new operator $b_{\xi, \eta, \lambda, \theta}^{(c)}$, to be referred to in the following as a bar operator, as follows:

$b_{\xi, \eta, \lambda, \theta}^{(c)}=\chi\left(c_{\xi, \eta, \lambda, \theta}-\alpha w_{\xi, \eta, \lambda, \theta}\right)$

where $\alpha$ is a constant and $\chi$ is the Heaviside step function $(\chi(z)=0$ for $z<0, \chi(z)=z$ for $z \geq 0)$. A similar model can be introduced for bar cells which use as input the computed responses of simple cells. In this case the complex cell response $c_{\xi, \eta, \lambda, \theta}$ has to be replaced by a simple cell response $s_{\xi, \eta, \lambda, \theta, \varphi}:$

$b_{\xi, \eta, \lambda, \theta, \varphi}^{(s)}=\chi\left(s_{\xi, \eta, \lambda, \theta, \varphi}-\alpha w_{\xi, \eta, \lambda, \theta}\right)$

Such a model is actually used for the illustrations given below.

${ }^{6}$ We presented the image shown in Fig. 12a to 20 people and asked them to describe briefly what they saw. In different words, all of them said that they saw two lines on a striped background. Three people said they saw 'two sides of a triangle' or 'a part of a triangle' - emphasising, however, that the triangle was not complete, having only two sides. Subsequently the same 20 people were presented with the two possible decompositions shown in Fig. 12c,d and Fig. 12e,f. All of them gave preference to the former decomposition, most of them completely rejecting the possibility of the latter one.
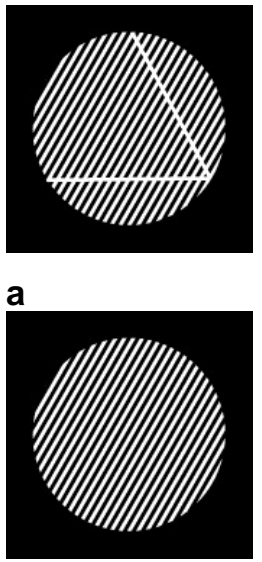

C

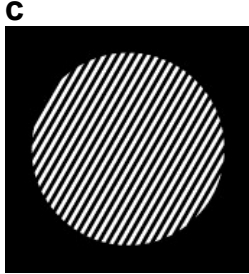

e

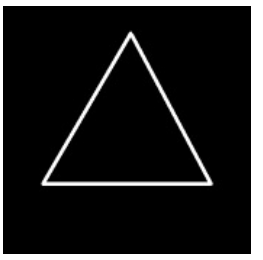

b

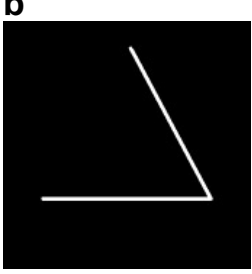

d

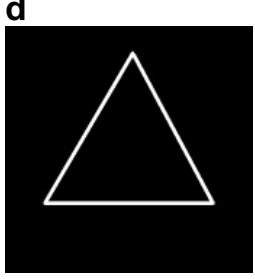

f
Fig. 12. The presence of a grating in the upper left image (a) suppresses the perception of a triangle in this image: while the two triangle sides which have orientations different from the orientation of the grating are clearly seen, the line segment which makes the third side of the triangle and has the same orientation as the grating is 'lost' in the grating. As illustrated by the upper right image (b), the triangle is well perceived if the other lines of the grating are removed. People are more likely to decompose the input image (a) into a grating and two lines $(\mathbf{c}, \mathbf{d})$ rather than a grating and a triangle $(\mathbf{e , f})$

If there is no texture at point $(\xi, \eta)$ and around it, i.e. $w_{\xi, \eta, \lambda, \theta}=0$, the outputs $b_{\xi, \eta, \lambda, \theta}^{(c)}$ and $b_{\xi, \eta, \lambda, \theta, \varphi}^{(s)}$ of these new operators are equal to the values $c_{\xi, \eta, \lambda, \theta}$ and $s_{\xi, \eta, \lambda, \theta, \varphi}$ of the corresponding complex and simple cell operators, respectively. In other words, if there is no texture the complex and simple cell activity caused, for instance, by a (single) bar is conveyed to the next stage of form processing:

$b_{\xi, \eta, \lambda, \theta}^{(c)}=c_{\xi, \eta, \lambda, \theta}, \quad b_{\xi, \eta, \lambda, \theta, \varphi}^{(s)}=s_{\xi, \eta, \lambda, \theta, \varphi}$

If, however, there is texture in the neighbourhood of the point and the activity of the grating cell operator is sufficiently strong, $\alpha w_{\xi, \eta, \lambda, \theta} \geq c_{\xi, \eta, \lambda, \theta}, \alpha w_{\xi, \eta, \lambda, \theta} \geq s_{\xi, \eta, \lambda, \theta, \varphi}$, no single-bar activity is conveyed to the next stage:

$b_{\xi, \eta, \lambda, \theta}^{(c)}=0, \quad b_{\xi, \eta, \lambda, \theta, \varphi}^{(s)}=0$

The bar operators introduced in this way will react to single bars but will not react to bars which make a part of a grating.

As already mentioned in Sect. 1, there is some neurophysiological evidence for the existence of cells which can be modelled by the above operators. Schiller et al. (1976), for instance, reported on cells in area V1 which reacted only to single bars but not at all to sine-wave gratings. Von der Heydt et al. (1992) also encountered this type of cells when looking for grating cells in the areas V1 and V2 of macaque monkeys.

An interesting experiment is described by Blakemore and Tobin (1972). They measured the response of a 'complex 


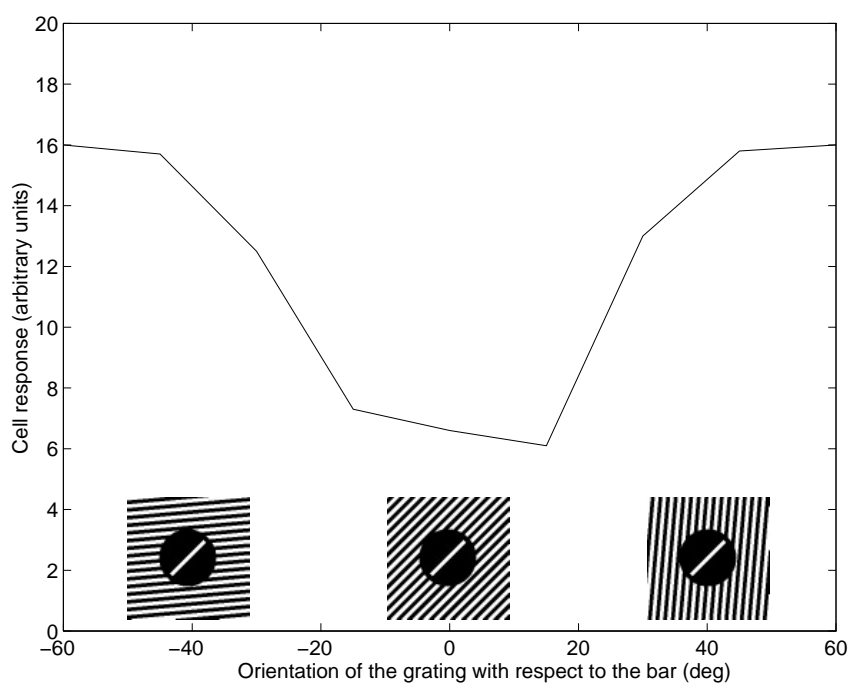

Fig. 13. The bar operator model introduced in (11) explains the results of an experiment described by Blakemore and Tobin (1972). They describe a 'complex cell' whose response to a bar with optimal size and orientation is inhibited by a grating pattern which covers the area outside the receptive field of the cell, defined as the region in which the cell reacts to a single bar stimulus. The inhibition strength depends on the difference between the orientation of the grating and the orientation of the optimal bar stimulus. The plot shown in the figure is obtained computationally by using (11). The resemblance to the curve actually measured by Blakemore and Tobin (1972) is amazing (compare with their Fig. 1)

cell' to a single bar stimulus which was surrounded by a grating pattern. First the position and size of the receptive field were estimated, together with the preferred orientation of the cell, using a single bar stimulus. The cell was classified as 'complex', because it showed unmodulated response to a moving bar. Next, a grating pattern was added which covered the entire visual field except for the area in which the cell responded to the bar stimulus. For a normal complex cell which complies with the complex cell model above, the addition of the grating should not have had any influence on the cell response. However, the cell response turned out to be inhibited by the surrounding grating. Apparently the receptive field of the cell was larger than was first concluded from the experiments with a single bar. The behaviour of this cell was evidently more complex than the behaviour of normal complex cells.

The bar operator model gives a good explanation of the observed phenomenon. The area in which a bar cell operator reacts to a single bar - which is the receptive field of the corresponding simple or complex cell - is smaller than the area in which a grating pattern can affect the cell response; the latter area is the receptive field of the corresponding grating cell.

Figure 13 shows the computed response of a bar cell operator to a stimulus that consists of a bar with optimal orientation and size and a grating pattern that surrounds the receptive field of the corresponding simple cell. The inhibition of the cell response is strongest when the orientation of the grating coincides with the orientation of the optimal bar stimulus. In the experiment of Blakemore and Tobin, the response of the cell was reduced to the level of spontaneous discharge activity of the cell. In our computer model the response is attenuated by a factor of 2.5 . When the deviation orientation difference is larger than $60^{\circ}$, there is no inhibition by the grating.

The bar operator model can in principle be extended by integration of the suppression term in (11) over a range of spatial frequencies:

$b_{\xi, \eta, \lambda, \theta}^{(c)}=\chi\left(c_{\xi, \eta, \lambda, \theta}-\int \alpha_{\lambda-\lambda^{\prime}} w_{\xi, \eta, \lambda^{\prime}, \theta} \mathrm{d} \lambda^{\prime}\right)$

In this case, the complex cell term $c_{\xi, \eta, \lambda, \theta}$ is inhibited not only by the grating cell term $w_{\xi, \eta, \lambda, \theta}$ which corresponds to the same main spatial frequency $1 / \lambda$, but also by similar terms $w_{\xi, \eta, \lambda^{\prime}, \theta}$ corresponding to other spatial frequencies. The plausibility of this extension can be tested by measuring the response of single-bar cells of the type described by Schiller et al. (1976) and von der Heydt et al. (1992) as a function of both the width of the bars and the (fundamental) frequency of the bar grating.

The computational model proposed above concerns the process of conveying or not conveying the activities of simple and complex cells to higher stages. We are deliberately not concerned with the possibility of negative feedback from grating cells to complex and simple cells since, as demonstrated elsewhere (Petkov et al. 1993), such interactions may radically change the impulse response of the computational model of simple cells and bring it in contradiction with the actually measured impulse responses of such cells.

\subsection{Biological role: selective detection of bars, lines and contours}

Figure 14 shows a set of feature images computed with various operators from the input image shown in Fig. 12a. The images in the first column of Fig. 14 are obtained by applying simple-cell operators ${ }^{7}$ of various orientations and the same preferred spatial frequency as the fundamental spatial frequency of the grating in the input image. The second column of Fig. 14 shows the feature images computed with the corresponding grating cell operators and the third column shows the feature images computed with the bar operators according to the model introduced in (12) above. While the simple cell operators detect all white bars, independent of whether they are isolated or make part of a periodic pattern, grating cell and bar operators are more selective, in that the former react only to periodic bar patterns and the latter only to bars which do not form part of a periodic structure. Figure 15 shows the superpositions of the images in each of the three columns of Fig. 14. The superpositions of grating and bar operators shown in Fig. 15c and Fig. 15d, respectively, can be generated also if these operators are applied to the images shown in Fig. 12c and Fig. 12d, respectively. In this way, the result of the application of grating and bar cell operators corresponds to the perceptually plausible decomposition of the input image into texture and form information (Fig. 12c,d).

A similar image to the one shown in Fig. 15a is an illusion taken from Kanizsa (1979), in which part of a rectangular contour line is occluded by a grating pattern (Fig. 16a).

\footnotetext{
${ }^{7}$ More precisely, symmetrical 'centre-on' operators $(\varphi=0)$ are used.
} 


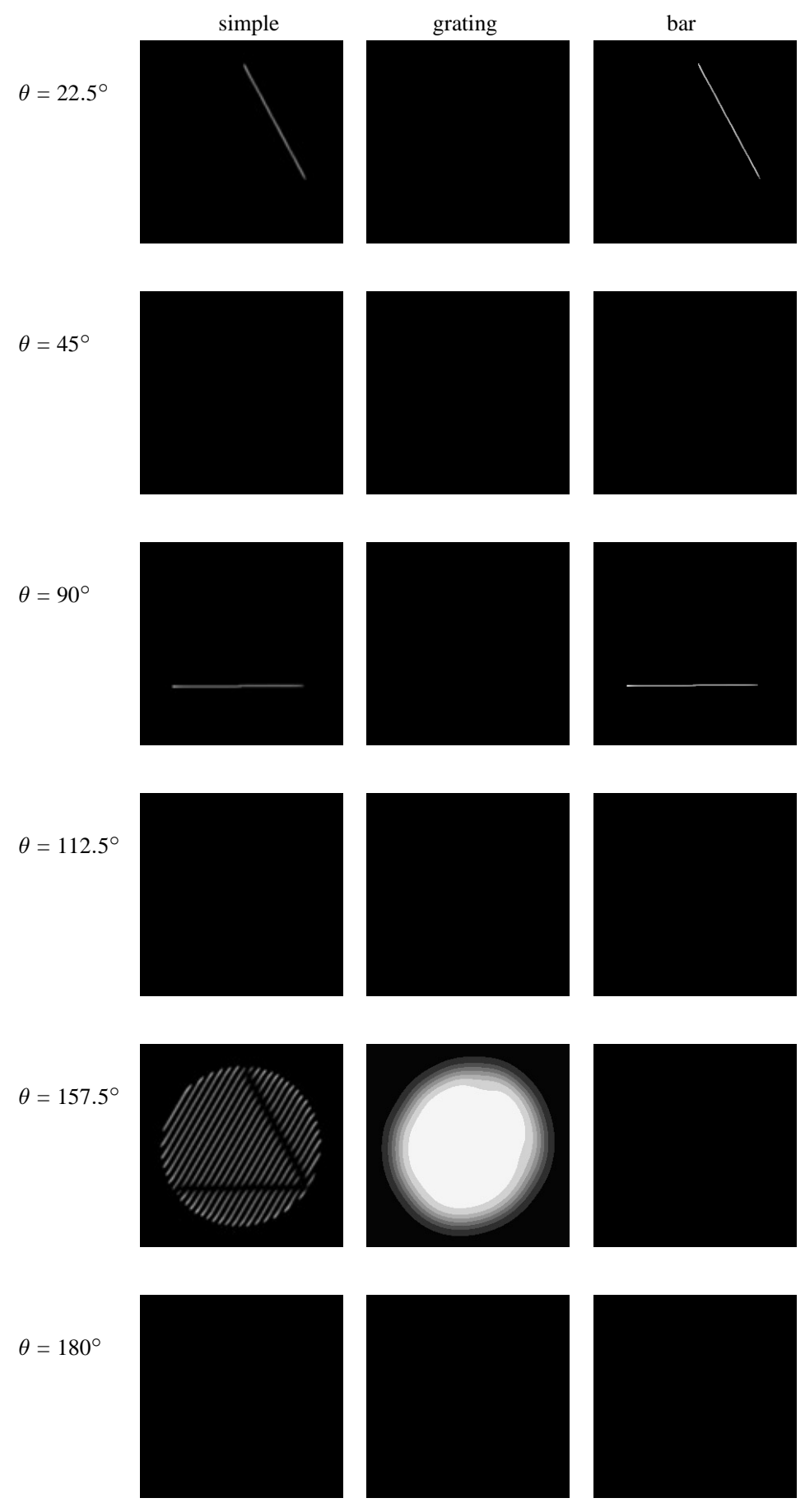

Fig. 14. Feature images computed from the input image shown in Fig.12a using simple cell, grating cell and bar cell operators of various orientations. Grating cell operators and bar cell operators, which react only to bar gratings and single bars, respectively, resolve the ambiguity of the features detected by simple cell operators, which react both to single bars and to gratings of bars
While simple cell operators (Fig. 16b) show an ambiguous response with respect to form and texture, the grating and bar cell operators are able to resolve this ambiguity (Fig. 16c,d).

The results of a computer simulation experiment with a natural image are shown in Fig. 17. The input image (Fig. 17a) shows a bottle standing on a table with a striped tablecloth. It was already shown that grating cell operators, in contrast to simple and complex cells operators, are able to detect the texture areas in the image, while they do not react to the contours of the bottle. The bar cell operator is complementary to the grating cell operator in that it reacts only to the contours of the bottle and not to texture. The combination of grating and bar cell operators gives visual information segregation which corresponds to the segregation inferred from psychophysical experiments.

\section{Summary and conclusions}

In this paper we introduced computational models of periodic- and aperiodic-pattern selective cells, called grating and bar cells, respectively, and applied them to different visual 


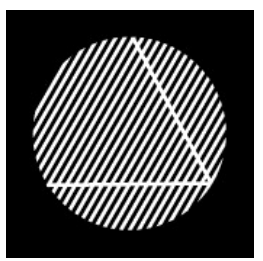

a

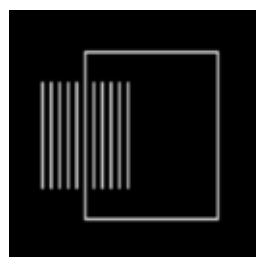

a

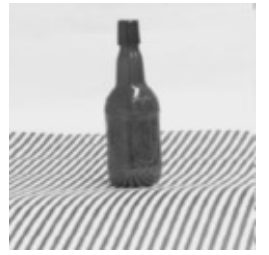

a

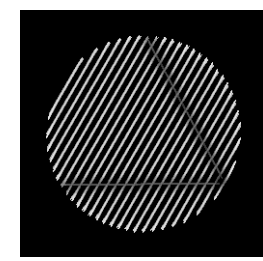

b

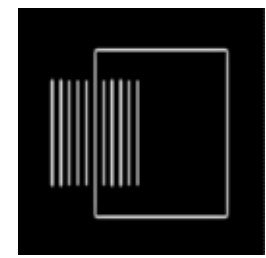

b

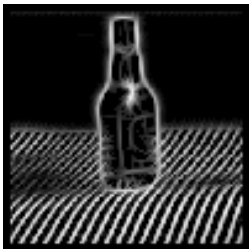

b

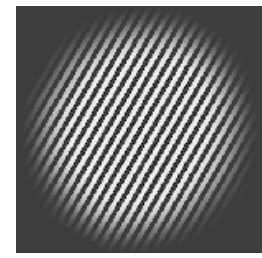

C

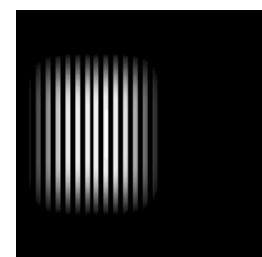

C

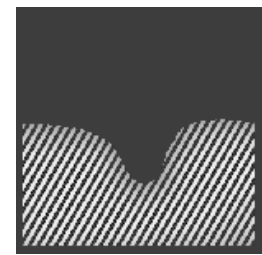

C

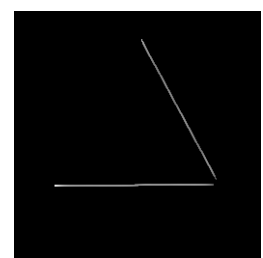

d

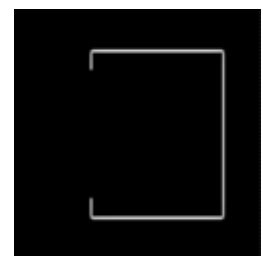

d

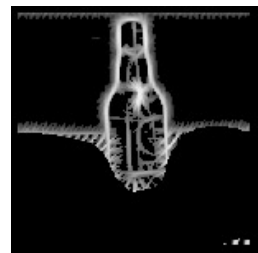

d
Fig. 15. Feature images computed as superpositions of feature images obtained with simple (b), grating (c) and bar (d) cell operators for different orientations. The ambiguity of simple cell responses for gratings and single bars is resolved by grating and bar cell operators. As regards the image shown in c, the actual grating cell feature image has been replaced by an image in which the region of activity of grating cells with given preferred orientation and periodicity is filled in with the optimal grating stimulus

Fig. 16. Another example of a grating pattern suppressing a contour line (a). The example (a) is taken from Kanizsa (1979). The feature images computed as superpositions of feature images obtained with simple cell, grating cell and bar operators are shown in b,c and $\mathbf{d}$, respectively

Fig. 17. An input image (a) and feature images computed as superpositions of feature images obtained with simple (b), grating (c) and bar (d) cell operators for different orientations stimuli in order to verify the models and reveal the biological role of the cells concerned.

The computational model of grating cells employs an AND-type non-linearity used to combine the responses of simple cells with symmetric receptive field profiles and opposite polarities in such a way that a grating cell will respond strongly to a bar grating of a given orientation and periodicity but will not react to single bars. The parameters of our model are chosen in such a way that all properties of grating cells, as reported in von der Heydt et al. (1992), are successfully mimicked. These properties range from orientation and spatial frequency bandwidths of such cells to their characteristic responses to selected aperiodic patterns such as isolated bars and edges, and periodic patterns such as gratings of different orientations and periodicities and checkerboard patterns.

As grating cell operators are selective for periodic oriented patterns, it was concluded by the neurophysiologists who discovered this type of cell that they play a definite role in the perception and processing of oriented texture at an early stage in the visual system. Since other orientationselective operators, such as simple and complex cell operators, have already been shown in computer simulations to be capable of detecting oriented texture, our main concern in this respect was what the added value of grating cells might be with respect to the perception and processing of texture.

Firstly, we demonstrated by means of computer simulations that grating cell operators succeed in detecting oriented texture where simple and complex cell operators do so. The different types of operators give comparable results for segmentation of different texture regions also. Then we illustrated the difference between simple and complex cell operators on the one hand, and grating cell operators on the other, by computer simulations in which the two types of operators are applied to input images which contain contours but not texture. In such cases simple and complex cell operators will give the wrong results if used as texture-detecting operators. They respond not only to texture but also to other image features such as edges, lines and contours. In contrast, grating cell operators detect no features such as isolated lines and edges. In this way grating cell operators fulfil a very important requirement imposed on texture-processing operators in that, in addition to successfully detecting (oriented) texture, they do not react to other image attributes such as object contours.

The difference between simple and complex cell operators, on the one hand, and grating cell operators, on the other, is especially well illustrated when these operators are applied to images which contain both texture and form information. While complex cell operators, for instance, detect both contours and texture and are, in this way, not capable of discriminating between these two different types of image features, grating cell operators detect exclusively (oriented) texture. We conclude that grating cell operators are more effective than simple and complex cell operators in the detection and processing of texture in that they are capable not only of detecting texture where it is present and also detected by simple or complex cell operators, but also of separating it from other image features such as edges and contours.

The computational model of a bar cell employs a thresholded difference of the activity of a complex or a simple cell and a grating cell with the same preferred orientation and spatial frequency. In the presence of oriented texture in the receptive fields of the cells concerned, in particular in 
the presence of a grating which regarding its orientation and periodicity is the optimal stimulus for that grating cell, the strong grating cell response will have a strong inhibitory effect on the response of the bar cell and eventually suppress its response completely. If there is no texture in the receptive fields of the cells concerned there will be no inhibitory effect from the grating cell and the bar cell will simply convey the response of the complex or simple cell.

This simple model is capable of qualitatively reproducing the main feature in the behaviour of bar cells, namely to respond to single bars and to decrease their responses with the addition of further bars to form a periodic pattern. Furthermore the model reproduces amazingly well the form of the response of such a cell as a function of the orientation of a grating which inhibits the response to an optimal single bar stimulus. The proposed model is also quite successful in explaining the effects of gratings on the perception of bars as these are known from psychophysical experiments.

The proposed model of bar cells is conceived in such a way that its response and the response of the related grating cell complement each other, in that their sum would produce the response of the corresponding complex or simple cell. In this way, a pair consisting of a grating cell and an associated bar cell carries the same information as the corresponding complex or simple cell. The role of this new representation of visual information is likely to be related to the efficient solution of specific visual tasks, in that such a representation makes certain features of the visual information, such as the presence of oriented texture or object contours, explicit, i.e. immediately accessible for interpretation without the need for further processing. This increasing functional specialisation in the transition of simple and complex cell activities to grating and bar cell activities seems to follow the same principles of increasing functional specialisation that are followed when the representation of visual information delivered by orientationally unselective retinal ganglion and lateral geniculate nucleus cells is transformed into a representation encoded in the activities of the orientationally selective simple and complex cells.

The question about the role of the transformation of visual information from a representation by simple and complex cell activities to bar and grating cell activities can be accessed only from the viewpoint of the goals of natural vision information processing. While the simple and complex cell operators detect bars, independent of whether these bars are isolated or form part of a periodic pattern, grating and bar cell operators are more selective, in that the former react only to periodic bar patterns and the latter only to bars which do not form part of a periodic structure. In this way the latter representation resolves the ambiguity of the former one with respect to the discrimination between important image features such as contours and texture. This representation explains the (psychophysical) observation that the perception of texture and the perception of form are complementary processes.

\section{References}

1. Albrecht DG, Thorell LG, De Valois RL (1979) Spatial and temporal properties of receptive fields in monkey and cat visual cortex. Soc Neurosci Abstr 9:775
2. Albrecht DG, De Valois RL, Thorell LG (1980) Visual cortical neurons: are bars or gratings the optimal stimuli? Science 207:88-90

3. Andrews BW, Pollen DA (1979) Relationship between spatial frequency selectivity and receptive field profile of simple cells, J Physiol (Lond) 287:163-176

4. Bergen JR, Landy MS (1991) Computational modelling of visual texture segregation. In: Landy MS, Movshon JA (eds) Computational models of visual processing. MIT Press, Cambridge, Mass, pp 253-271

5. Blakemore C, Tobin EA (1972) Lateral inhibition between orientation detectors in the cat's visual cortex. Exp Brain Res 15:439-440

6. Burr DC, Morrone MC, Spinelli D (1989) Evidence for edge and bar detectors in human vision. Vision Res 29:419-431

7. Daugman JG (1985) Uncertainty relations for resolution in space, spatial frequency, and orientation optimized by two-dimensional visual cortical filters, J Opt Soc Am A 2:1160-1169

8. De Valois RL, Albrecht DG, Thorell LG (1978) Cortical cells: bar and edge detectors, or spatial frequency filters. In: Cool SJ, Smith EL III (eds) Frontiers of visual science, Springer, Berlin Heidelberg New York

9. De Valois KK, De Valois RL, Yund EW (1979) Responses of striate cortical cells to grating and checkerboard patterns. J Physiol (Lond) 291:483-505

10. De Valois RL, Albrecht DG, Thorell LG (1982) Spatial frequency selectivity of cells in macaque visual cortex. Vision Res 22:545-559

11. Field DJ, Nachmias J (1984) Phase reversal discrimination, Vision Res 24:333-340

12. Field DJ, Tolhurst DJ (1986) The structure and symmetry of simple cell receptive field profiles in the cat's visual cortex, Proc R Soc Lond B 228:379-399

13. Glezer VD, Tscherbach TA, Gauselman VE, Bondarko VM (1980) Linear and non-linear properties of simple and complex receptive fields in area 17 of the cat visual cortex, Biol Cybern 37:195-208

14. Hubel DH, Wiesel T (1962) Receptive fields, binocular interaction, and functional architecture in the cat's visual cortex. J Physiol (Lond) 160:106-154

15. Hubel DH, Wiesel TN (1974) Sequence regularity and geometry of orientation columns in the monkey striate cortex. J Comp Neurol $158: 267-293$

16. Hubel DH (1982) Explorations of the primary visual cortex, 1955-1978 (1981 Nobel Prize lecture). Nature 299:515-524

17. Jones JP, Palmer LA (1987) An evaluation of the two-dimensional Gabor filter model of simple receptive fields in cat striate cortex. J Neurophysiol 58:1233-1258

18. Kanizsa G (1979): Organization in Vision, Essays on gestalt perception. Praeger, New York

19. Kruizinga P, Petkov N (1995) A computational model of periodicpattern-selective cells. In: Mira J, Sandoval F (eds) From natural to artificial neural computation. Proc IWANN '95. (Lecture Notes in Computer Science, vol 930) Springer, Berlin Heidelberg New York, pp 90-99

20. Kulikowski JJ, Bishop PO (1981) Fourier analysis and spatial representation in the visual cortex, Experientia 37:160-163

21. Macleod IDG, Rosenfeld A (1974) The visibility of gratings: spatial frequency channels or bar-detecting units? Vision Res 14:909-915

22. Maffei L, Morrone C, Pirchio M, Sandini G (1979) Responses of visual cortical cells to periodic and non-periodic stimuli. J Physiol (Lond) 296:27-47

23. Malik J, Perona P (1990) Preattentive texture discrimination with early vision mechanisms. J Opt Soc 7:923-932

24. Manjunath BS, Chellappa R (1993) A unified approach to boundary perception: edges, textures, and illusory contours. IEEE Trans Neural Networks 4:96-107

25. Marcelja S (1980) Mathematical description of the responses of simple cortical cells. J Opt Soc Am 70:1297-1300

26. Morrone MC, Burr DC (1988) Feature detection in human vision: Aphase-dependent energy model. Proc R Soc Lond B 235:221-245

27. Movshon JA, Thompson ID, Tolhurst DJ (1978) Spatial summation in the receptive fields of simple cells in the cat's striate cortex, J Physiol (Lond) 283:53-77

28. Movshon JA, Thompson ID, Tolhurst DJ (1978) Receptive field organisation of complex cells in the cat's striate cortex. J Physiol (Lond) 
283:79-99

29. Petkov N, Kruizinga P, Lourens T (1993) Orientation competition in cortical filters: an application to face recognition. Computing Science in The Netherlands 1993. Stichting Mathematisch Centrum, Amsterdam, pp 285-296

30. Petkov N (1995) Biologically motivated computationally intensive approaches to image pattern recognition. Future Generation Comput Syst 11:451-465

31. Pollen D, Ronner S (1981) Phase relationships between adjacent simple cells in the visual cortex. Science 212:1409-1411

32. Schiller PH, Finlay BL, Volman SF (1976) Quantitative studies of single-cell properties in monkey striate cortex. III. Spatial frequencies, J Neurophysiol 39:1334-1351

33. Shapley R, Caelli T, Morgan M, Rentschler I (1990) Computational theories of visual perception. In: Spillmann L, Werner JS (eds) Visual Perception: the neurophysiological foundations. Academic Press, New York, pp 417-448
34. Spitzer H, Hochstein S (1985) A complex cell receptive field model. J Neurophysiol 53:1266-1286

35. Szulborski RG, Palmer LA (1990) The two-dimensional spatial structure of non-linear subunits in the receptive fields of complex cells. Vision Res 30:249-254

36. Tyler CW (1978) Selectivity for spatial frequency and bar width in cat visual cortex. Vision Res 18:121-122

37. von der Heydt R (1987) Approaches to visual cortical function. Rev Physiol Biochem Pharmacol 108:69-150

38. von der Heydt R, Peterhans E, Dürsteler MR (1991) Grating cells in monkey visual cortex: Coding texture. In: Blum B (ed) Channels in the visual nervous system: neurophysiology, psychophysics and models. Freund, London, pp 53-73

39. von der Heydt R, Peterhans E, Dürsteler MR (1992) Periodic-patternselective cells in monkey visual cortex. J. Neurosci 12:1416-1434 